\title{
Autoantibody binding in liquid phase to IL-2 in human sera is not type 1 diabetes specific
}

\author{
Ilaria Marzinotto ${ }^{1}$ - Daniela Liberati ${ }^{2} \cdot$ Cristina Brigatti $^{1} \cdot$ Riccardo Bonfanti $^{3}$. \\ Angela Stabilini ${ }^{1}$ Paolo Monti ${ }^{1}$. Emanuele Bosi ${ }^{1,4} \cdot$ Lorenzo Piemonti $^{1,4}$. \\ Vito Lampasona ${ }^{2}$
}

Received: 30 May 2017 / Accepted: 13 June 2017 / Published online: 19 July 2017

(C) Springer-Verlag GmbH Germany 2017

Keywords Autoantibodies · Autoimmunity · ELISA · IL-2 · Immunoprecipitation · LIPS · Type 1 diabetes

\author{
Abbreviations \\ LIPS Luciferase immunoprecipitation system \\ NLuc-IL-2 Recombinant IL-2 tagged with a NanoLuc \\ luciferase reporter \\ RBA Radiobinding assay \\ ROC-AUC Area under the receiver operator characteristic \\ curve
}

To the Editor: Autoantibodies against pancreatic islet antigens are major biomarkers of type 1 diabetes and have found widespread application in both diagnostics and research settings. Known type 1 diabetes autoantigens targeted by antibody responses include proteins linked to either the insulin or neurotransmitter secretory pathways of pancreatic beta cells. Recently, the presence of autoantibodies against the cytokine IL-2 was reported in individuals with type 1 diabetes [1]. Autoantibodies against other cytokines, such as IFN- $\gamma$, granu-

Vito Lampasona

lampasona.vito@hsr.it

1 Diabetes Research Institute, IRCCS San Raffaele Scientific Institute, Milan, Italy

2 Division of Genetics and Cell Biology, IRCCS San Raffaele Scientific Institute, Via Olgettina 60, 20132 Milan, Italy

3 Department of Pediatrics, IRCCS San Raffaele Scientific Institute, Milan, Italy

4 Vita-Salute University, Milan, Italy locyte colony-stimulating factor, IL-6, IL-17 and IL-22, have been described in a variety of other diseases, including autoimmune syndromes sometimes presenting together with type 1 diabetes [2]. In an original report by Pérol et al [1], IL-2 autoantibodies, measured by a direct ELISA assay, were suggested to be present in the NOD mouse, where they correlated with disease development, and in around $25 \%$ of humans at onset of type 1 diabetes. To investigate the presence of IL- 2 autoantibodies in human type 1 diabetes, we developed a novel assay based on the luciferase immunoprecipitation system (LIPS) format. The LIPS is a derivative of the radiobinding assay (RBA), the gold standard method for the measurement of type 1 diabetes-associated autoantibodies, in which serum antibodies are used to immunoprecipitate radiolabelled antigens in liquid phase. In the LIPS, the use of radiolabelled tracers is superseded in favour of chimeric recombinant antigens tagged with a luciferase reporter [3]. We generated a recombinant IL-2 (NLuc-IL-2) fused to a NanoLuc luciferase reporter (Promega, Madison, WI, USA) and expressed NLuc-IL-2 in mammalian eukaryotic cells as a protein secreted in the culture medium at a concentration of $204 \mathrm{ng} / \mathrm{ml}$, as quantified using a commercially available sandwich IL-2 ELISA kit. The biological activity of NLuc-IL-2 was verified by flow cytometry using a carboxyfluorescein succinimidyl ester (CFSE)-based cell proliferation assay. We incubated $6 \times 10^{5}$ cells from a buffy coat preparation for 1 week and observed a proliferation in 52\% of lymphocytes treated with $0.7 \mathrm{ng}$ of NLuc-IL-2 vs $11 \%$ in untreated cells and $>62 \%$ in cells treated with commercial recombinant human IL-2. Using the LIPS, we then measured IL-2 autoantibodies in serum from individuals with new-onset type 1 diabetes ( $n=60$, median age 13.9 years [interquartile range 7.914.9 years]), diagnosed according to the ADA criteria, and from control individuals without diabetes $(n=61$, median age 22.1 years [interquartile range 16.7-26.1 years]) collected at the San Raffaele Scientific Institute between 2012 and 2016 within 


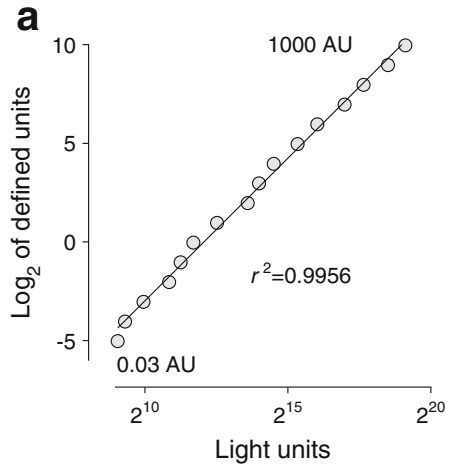

Fig. 1 Anti-IL-2 autoantibody titres measured by LIPS. (a) Standard curve of serial twofold dilutions of the anti-human IL-2 monoclonal antibody MQ1-17H12 used to convert raw data in light units into arbitrary units (AU) in the LIPS assay. Values in AU attributed to the lowest and highest points of the curve are shown. (b) Scatter plot of anti-IL-2 titres on

the study protocol TIGET004-DRI003. The study was approved by the local ethics committee, all participants gave informed consent and the investigations were carried out in accordance with the Declaration of Helsinki as revised in 2000. Serial twofold dilutions of the anti-human IL-2 monoclonal antibody MQ1-17H12 [1], ranging from 1:1000 to $1: 6,348,000$, were used to build a standard curve and convert raw results in arbitrary units (Fig. 1a). Our results show that binding to NLuc-IL-2 could not be detected in any of the tested type 1 diabetes samples above the adopted threshold for positivity, corresponding to the 99th percentile of values observed in control samples as commonly adopted in RBA for other type 1 diabetes-associated autoantibodies (Fig. 1b). The analysis of the area under the receiver operator characteristic curve (ROC-AUC) confirmed that the measurement of IL-2 antibodies in a liquid phase assay could not discriminate between individuals with and without type 1 diabetes (anti-IL-2 LIPS ROC-AUC 0.537 [95\% CI 0.4341, 0.6405], $p=0.479$ ). The size of the cohorts analysed provided a $>80 \%$ power to detect a difference in the proportion of IL-2 autoantibodypositive individuals in the two groups as large as the one reported in previous studies. The use of commercial recombinant IL-2 to compete for binding to NLuc-IL-2 in LIPS of sera with the highest signal again did not discriminate between samples from individuals with and without diabetes. Therefore, we cannot confirm the original findings of the presence of anti-IL-2 autoantibodies in type 1 diabetes claimed by Pérol et al [1] and based on a solid-phase immunoassay. Several possible mechanistic explanations have been previously advanced to explain the lack of concordance between liquid-phase and solid-phase assay results [4]. However, it is important to note that, more than once, liquid-phase assays have in the past outperformed classical solid-phase assays for measurement of type 1 diabetes autoantibody responses [5]. In conclusion, our results do not support the presence of IL-2 autoantibodies or their relevance for the pathogenesis of human type 1 diabetes. a $\log _{2}$ scale, measured by LIPS in sera from individuals with newly diagnosed type 1 diabetes $(n=60)$ and from control individuals without diabetes $(n=61)$. The dashed line represents the threshold for positivity placed at the 99th percentile of values observed in control samples

Acknowledgements We wish to thank the BioBank of the Immunohematology and Transfusion Medicine Unit, led by L. Santoleri at the San Raffaele Scientific Institute, Milan, Italy, for their assistance in sample collection and retrieval.

Data availability Data generated in this study are available from the authors upon request.

Funding This study was conducted within the framework of the Italian Ministry of Research's project 'Ivascomar project, Cluster Tecnologico Nazionale Scienze della Vita ALISEI'.

Duality of interest The authors declare that there is no duality of interest associated with this manuscript.

Contribution statement IM, DL, CB, RB, AS and PM acquired and analysed the data. EB, LP and VL designed the study, analysed the data and wrote the manuscript. All authors revised and approved the final version of the manuscript. VL is responsible for the integrity of the work as a whole.

\section{References}

1. Pérol L, Lindner JM, Caudana P et al (2016) Loss of immune tolerance to IL-2 in type 1 diabetes. Nat Commun 7:13027

2. Browne SK (2014) Anticytokine autoantibody-associated immunodeficiency. Annu Rev Immunol 32:635-657

3. Burbelo PD, Hirai H, Leahy $\mathrm{H}$ et al (2008) A new luminescence assay for autoantibodies to mammalian cell-prepared insulinomaassociated protein 2. Diabetes Care 31:1824-1826

4. Lampasona V, Ferrari M, Bosi E et al (1994) Sera from patients with IDDM and healthy individuals have antibodies to ICA69 on western blots but do not immunoprecipitate liquid phase antigen. J Autoimmun 7:665-674

5. Liu E, Eisenbarth GS (2007) Accepting clocks that tell time poorly: fluid-phase versus standard ELISA autoantibody assays. Clin Immunol 125:120-126 